\title{
Using DOProC method in reliability assessment of steel elements exposed to fatigue
}

\author{
Martin Krejsa ${ }^{1, *}$, Jiri Brozovsky ${ }^{1}$, David Mikolasek ${ }^{1}$, Petr Lehner $^{1}$ and Premysl Parenica ${ }^{2}$ \\ ${ }^{1}$ VŠB-Technical University of Ostrava, Faculty of Civil Engineering, Department of Structural \\ Mechanics, Ludvika Podeste 1875/17, 70833 Ostrava - Poruba, Czech Republic \\ ${ }^{2}$ VŠB-Technical University of Ostrava, Faculty of Civil Engineering, Department of Building \\ Structures, Ludvika Podeste 1875/17, 70833 Ostrava - Poruba, Czech Republic
}

\begin{abstract}
Fatigue crack damage depends on a number of stress range cycles. This is a time factor in the course of reliability for the entire designed service life. Three sizes are important for the characteristics of the propagation of fatigue cracks - initial size, detectable size and acceptable size. The theoretical model of fatigue crack progression can be based on a linear fracture mechanic. Depending on location of an initial crack, the crack may propagate in structural element e.g. from the edge or from the surface. When determining the required degree of reliability, it is possible to specify the time of the first inspection of the construction which will focus on the fatigue damage. Using a conditional probability and Bayesian approach, times for subsequent inspections can be determined. For probabilistic modelling of fatigue crack progression was used the original and new probabilistic method - the Direct Optimized Probabilistic Calculation ("DOProC"), which uses a purely numerical approach without any simulation techniques or approximation approach based on optimized numerical integration.
\end{abstract}

\section{Introduction to probabilistic computation}

In process of designing a structure under static or dynamic loads [1], various reliability criteria should be met in accordance with the relevant standards. Common studies of the misalignment of failure probability $P_{f}$ (or the reliability index $\beta$ ) over time tend to focus only on structural details, however, a comprehensive probabilistic methodology generally applicable to bridge structures is currently missing. Numerous stochastic methods, which enable the determination of failure probability, respectively the reliability index, have been developed [2]. A substantial part of these methods are based on the crude Monte Carlo simulation method (MC), whose disadvantage is poor efficiency due to the need of a high number of simulation steps. Advanced and stratified simulation methods, for e.g. Markov chain Monte Carlo simulations (MCMC), also applied in fatigue damage prognosis, strive to increase the efficiency of these computational methods. An alternative solution is the evaluation of reliability using the reliability index, which can be efficiently determined using the Latin Hypercube Sampling method (LHS). The LHS method is capable of

* Corresponding author: martin.krejsa@,vsb.cz 
evaluating the reliability index from a small number of simulation steps (hundreds to thousands).

Another category of stochastic approaches for the quantification of the reliability of structures are represented by methods, which determine the failure probability on the basis of numerical integration, for e.g. the Direct Optimized Probabilistic Calculation method DOProC, which was comprehensively published, e.g. in [3, 4]. It appears that this method is very effective for many probabilistic problems. It is also distinguished by higher accuracy than that of simulation methods, resp. approximation ones. This calculation method was applied in the solution of some engineering problems, among others, the assessment of reliability of steel bridge structures loaded by fatigue [5]. Probabilistic modelling of fatigue crack progression leads to designing a system of regular inspections of structures [6].

\section{Fatigue crack propagation using linear fracture propagation}

When investigating into the propagation, the fatigue crack that deteriorates a certain area of the structure components is described with one dimension only $a$ [7]. In order to describe the propagation of the crack, the linear elastic fracture mechanics is typically used. This method defines the limit of propagation rate of the crack $(\mathrm{d} a / \mathrm{d} N)$ and range of the stress rate coefficient in the face of the crack using the Paris-Erdogan law [8]:

$$
\frac{\mathrm{d} a}{\mathrm{~d} N}=C \cdot \Delta K^{m}
$$

where $C, m$ are material constants, that are determined experimentally [9], $a$ is the crack size, $N$ is the number of loading cycles and $\Delta K$ is the stress rate coefficient. The fatigue crack will propagate in a stable way only if the initial crack $a_{0}$ exists in the place where the stress is concentrated. This place is located, e.g., at the edge or on the surface of the element.

Having modified (1) according [5], the following formula will be achieved:

$$
\int_{a_{1}}^{a_{2}} \frac{\mathrm{d} a}{\left(\sqrt{\pi \cdot a} \cdot F_{(a)}\right)^{m}}=\int_{N_{1}}^{N_{2}} C \cdot \Delta \sigma^{m} \cdot \mathrm{d} N
$$

where number of cycles from $N_{1}$ to $N_{2}$ is needed to increase the crack from the $a_{1}$ to $a_{2}$, $\Delta \sigma$ stress range, and $F_{(a)}$ is the calibration function which represents the course of propagation of the crack. Depending on location of an initial crack, the crack may propagate from the edge or from the surface [10].

\section{Probabilistic reliability assessment}

Three sizes are important for the characteristics of the propagation of fatigue cracks. The first size is the initiation size of the crack $a_{0}$ that corresponds to a random failure in an element subject to random loads. Existence of the initiation cracks during the propagation should be revealed, along the detectable length of the crack $a_{d}$, during inspections. The crack propagates in a stable way until it reaches the third important size - acceptable length of the crack $a_{a c}$, which is a limit for the required reliability.

The primary assumption is that the primary design should take into account the effects of the extreme loading and the fatigue resistance should be assessed then. This means, the safety margin is defined e.g. by: 


$$
g(R, E)=G=R-E,
$$

where $R$ is the random resistance of the element and $E$ represents random variable effects of the extreme load.

The probabilistic methods should be used for the investigation into the propagation rate of the fatigue crack until the acceptable size is reached because the input variables include uncertainties and reliability should be taken into account. If the length of the crack length $a_{1}$ in (2) equals to the initial length $a_{0}$ and if $a_{2}$ in (2) equals to the acceptable crack length $a_{a c}$, the left-hand side of (2) can be regarded as the resistance of the structure - $R$ :

$$
R_{\left(a_{a c}\right)}=\int_{a_{0}}^{a_{a c}} \frac{\mathrm{d} a}{\left(\sqrt{\pi \cdot a} \cdot F_{(a)}\right)^{m}} .
$$

Similarly, it is possible to define the cumulated effect of loads that is equal to the right side (randomly variable effects of the extreme load) of (2):

$$
E_{(N)}=\int_{N_{0}}^{N} C \cdot \Delta \sigma^{m} \cdot \mathrm{d} N=C \cdot \Delta \sigma^{m} \cdot\left(N-N_{0}\right),
$$

where $N$ is the total number of oscillations of stress peaks $(\Delta \sigma)$ for the change of the length from $a_{0}$ to $a_{a c}$, and $N_{0}$ is the number of oscillations in the time of initialisation of the fatigue crack (typically, the number of oscillations is zero).

It is possible to define a reliability function $G_{\text {fail }}$ :

$$
G_{f a i l(\mathbf{x})}=R_{\left(a_{a c}\right)}-E_{(N)},
$$

where $\mathbf{X}$ is a vector of random physical properties such as mechanical properties, geometry of the structure, load effects and dimensions of the fatigue crack.

The analysis of the reliability function (6) gives a failure probability $P_{f}$, which equals to:

$$
P_{f}=P\left(G_{f a i l(\mathbf{x})}<0\right)=P\left(R_{\left(a_{a c}\right)}-E_{(N)}<0\right) .
$$

\section{Design of structural inspection system}

Because it is not certain in the probabilistic calculation whether the initiation crack exists and what the initiation crack size is and because other inaccuracies influence the calculation of the crack propagation, a specialised inspection is necessary to check the size of the measureable crack in a specific period of time. The acceptable crack size influences the time of the inspection. If no fatigue cracks are found, the analyses of inspection results give conditional probability during occurrence.

While the fatigue crack is propagating, it is possible to define following random phenomena that are related to the growth of the fatigue crack and may occur in any time, $t$, during the service life of the structure:

$\boldsymbol{U}_{(t)}$ phenomenon: No fatigue crack failure has not been revealed within the $t$-time and the fatigue crack size $a_{(t)}$ has not reached the detectable crack size, $a_{d}$. This means:

$$
a_{(t)}<a_{d}
$$

$\boldsymbol{D}_{(t)}$ phenomenon: A fatigue crack failure has been revealed within the $t$-time and the fatigue crack size $a_{(t)}$ is still below the acceptable crack size $a_{a c}$. This means: 


$$
a_{d} \leq a_{(t)}<a_{a c}
$$

$F_{(t)}$ phenomenon: A failure has been revealed within the $t$-time and the fatigue crack size $a_{(t)}$ has reached the acceptable crack size $a_{a c}$. This means:

$$
a_{(t)} \geq a_{a c}
$$

If the crack is not revealed within the $t$-time, this may mean that there is not any fatigue crack in the structural element. This might be also an initiative phase of nucleation of the fatigue crack (when a crack appears in the material) and this phenomenon is not taken into account in the fracture mechanics. Even if the fatigue crack is not revealed it is likely that it exists there but the fatigue crack size is so small that it cannot be detected under existing conditions.

Using the phenomena above, it is possible to define following probabilities:

The probability that the failure is not detected within the $t$-time, this means the probability that the fatigue crack size $\mathrm{a}_{(t)}$ is below the measurable crack size $a_{d}$ :

$$
P\left(U_{(t)}\right)=P\left(a_{(t)}<a_{d}\right) .
$$

The probability that the failure detected within the $t$-time has the crack size $a_{(t)}$ that is less than the acceptable size $a_{a c}$ :

$$
P\left(D_{(t)}\right)=P\left(a_{d} \leq a_{(t)}<a_{a c}\right) .
$$

The probability that the failure occurs within the $t$-time, this means the probability that the fatigue crack size $a_{(t)}$ reaches the acceptable size $a_{a c}$ :

$$
P\left(F_{(t)}\right)=P\left(a_{a c} \leq a_{(t)}\right) .
$$

Those three phenomena cover the complete spectrum of phenomena that might occur in the $t$-time. This means:

$$
P\left(U_{(t)}\right)+P\left(D_{(t)}\right)+P\left(F_{(t)}\right)=1 .
$$

The probable course of the growth of the fatigue crack is shown in Fig. 1. The probabilities (11) - (13) can be determined in any period of time, $t$, using the probabilistic methods. The probabilistic calculation is carried out in time steps where one step typically equals to one year of the service life of the structure. When the probability of failure $P\left(F_{(t)}\right)$ reaches the designed failure probability $P_{d}$ [11], an inspection should be carried out in order to find out fatigue cracks, if any, in the structural element. The inspection provides information about conditions of the structure. Such conditions can be taken into account when carrying out further probabilistic calculations. The inspection in the $t$ time may result in any of the three mentioned phenomena.

Using the inspection results for the $\mathrm{t}$ time, it is possible to define the probability of the mentioned phenomena in times $T>t_{I}$. For that purpose, the conditional probability should be taken into consideration. If the event of interest is $A$ and the event $B$ is known or assumed to have occurred, probability of $A$ under the condition $B$ is:

$$
P(A \mid B)=\frac{P(A \cap B)}{P(B)}, \text { where } P(B)>0 .
$$




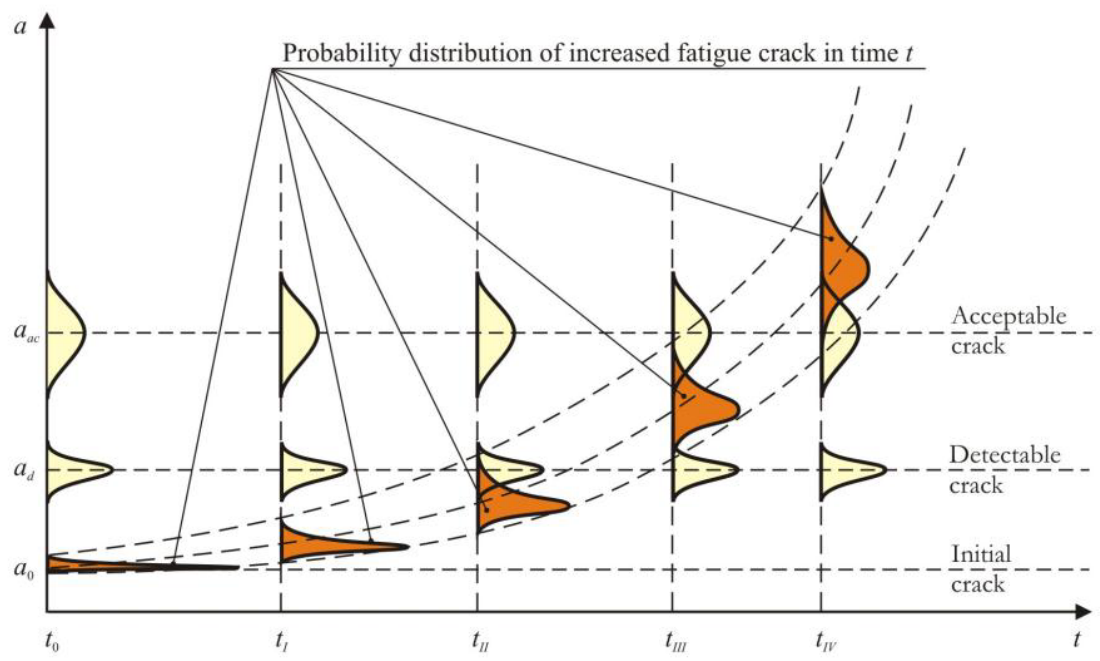

Fig. 1. Probabilistic growth of the fatigue crack in the course of time $t$.

In order to specify the time for the next inspection, it is necessary to determine the conditioned probabilities which can be expressed using the full probability rule:

$$
P(A)=P\left(H_{1}\right) \cdot P\left(A \mid H_{1}\right)+P\left(H_{2}\right) \cdot P\left(A \mid H_{2}\right)+\ldots+P\left(H_{n}\right) \cdot P\left(A \mid H_{n}\right),
$$

where $H_{1}, H_{2}, \ldots H_{n}$ is a system of mutually incompatible phenomena, and $A$ is the random event, which can occur simultaneously only with any of the phenomena $H_{i}$. For the probabilistic design of structural inspection system the phenomena $H_{1}, H_{2}, \ldots H_{n}$ represent events $U, D$ and $F$. Bayes' theorem describes the probability of an event, based on prior knowledge of conditions that might be related to the selected event:

$$
P(A \mid B)=\frac{P(B \mid A) \cdot P(A)}{P(B)}, \text { where } P(B)>0 .
$$

In (17) $A$ and $B$ are random events under analysis, $P(A)$ and $P(B)$ are the probabilities of observing $A$ and $B$ without regard to each other, $P(A \mid B)$ is a conditional probability according (15), which define the probability of observing event $A$ given that $B$ is true, and $P(B \mid A)$ is the probability of observing event $B$ given that $A$ is true.

In the Bayesian (or epistemological) interpretation, probability measures a "degree of belief." Bayes' theorem then links the degree of belief in a proposition before and after accounting for evidence. For proposition $A$ and evidence $B, P(A)$ - the "prior", is the initial degree of belief in $A, P(A \mid B)$ - the "posterior", is the degree of belief having accounted for $B$ and the quotient $P(B \mid A) / P(B)$ represents the support $B$ provides for $A$.

With these findings it can be defined the probability of event $F$ in time $T$ with respect to the results of structural inspection in time $t_{I}$ :

$$
P\left(F_{(T)} \mid U_{\left(t_{I}\right)}\right)=\frac{P\left(F_{(T)}\right)-P\left(F_{\left(t_{I}\right)}\right)-P\left(D_{\left(t_{I}\right)}\right) \cdot P\left(F_{(T)} \mid D_{\left(t_{I}\right)}\right)}{P\left(U_{\left(t_{I}\right)}\right)}
$$

and 


$$
P\left(F_{(T)} \mid D_{\left(t_{I}\right)}\right)=\frac{P\left(F_{(T)}\right)-P\left(F_{\left(t_{I}\right)}\right)-P\left(U_{\left(t_{I}\right)}\right) \cdot P\left(F_{(T)} \mid U_{\left(t_{I}\right)}\right)}{P\left(D_{\left(t_{I}\right)}\right)} .
$$

If re-distribution of stress from a point that is weakened by the crack is not taken into account, the crack propagation crack is usually rather high in the practical range of detectable values. If a fatigue crack is found during the inspection, it is necessary to monitor the safe growth of the crack or to take actions that will slow down or stop further propagation of the fatigue crack. In order to time the inspections well, (18) is most important. It defines the failure probability in $T>t_{\mathrm{I}}$ provided that no fatigue cracks have been revealed during the last inspection. It is clear from the equation that the results of the failure probability are influenced by mutual relations between the three crack sizes - the initial crack size $a_{0}$, measurable crack size $a_{d}$ and acceptable crack size $a_{a c}$.

The probabilities in (18) can be calculated in any $T>t_{\mathrm{I}}$ time using probabilistic methods. When the failure probability (18) reaches the designed failure probability $P_{d}$, an inspection should be carried out in order to reveal fatigue cracks, if any, in the construction component. The inspection may result in one of the three mentioned phenomena with corresponding probabilities. The entire calculation can be repeated in order to ensure welltimed inspections in the future.

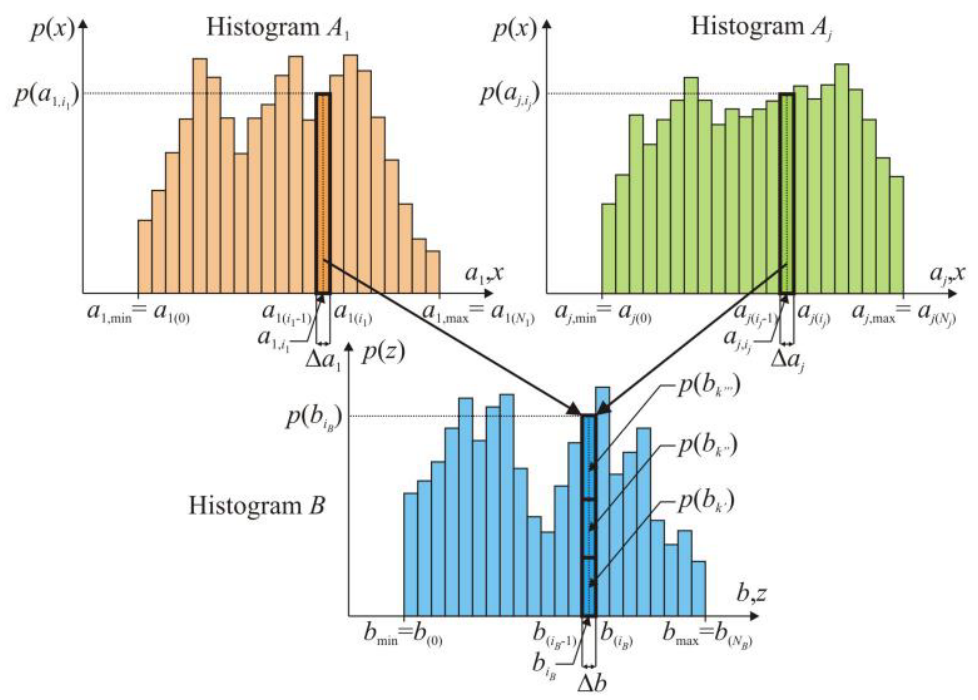

Fig. 2. Principle of the performing the numerical operations with the histograms of two random variables, which is similar to computation of structural resistance $R$ (4) or load effect $E$ (5).

\section{Probabilistic computation using DOProC method}

The basic computation algorithm of DOProC is based on general terms and procedures used in probabilistic theories. Let the histogram $B$ be an arbitrary function $f$ of histograms $A_{j}$ where $j$ ranges from 1 to $n$. Then:

$$
B=f\left(A_{1}, A_{2}, A_{3}, \ldots, A_{j}, \ldots A_{n}\right) .
$$


Each histogram $A_{j}$ consists of $i_{j}$ interval, where $i_{j}$ ranges from 1 to $N_{j}$. Performing these numerical operations of the probabilistic calculation with two random variables $A_{1}$ and $A_{j}$ expressed by histograms is shown in scheme in the Fig. 2.

The number of intervals $i_{j}$ in each histogram of the random variables $A_{j}$ can be similar as the number of $i_{B}$ intervals in the histogram of the resulting random variable $B$. The number of intervals is very important for the total number of needed numerical operations and required computing time. On top of this, the accuracy of the calculation depends considerably on the number of intervals. If there are too many random quantities, the tasks require too much time even if advanced computational facilities are available. Therefore, efforts have been made to optimize calculations in order to reduce the number of operations, while retaining reliable calculation results (for details see [3]).

Using this probabilistic approach is possible to determine the probabilities of random events $U, D$ and $F$ according (11) - (13), and, of course, using conditional probability (18) and the time of the designed failure probability $P_{d}$ the time of the inspections focused on the structural elements susceptible to fatigue damage (see Fig. 3).

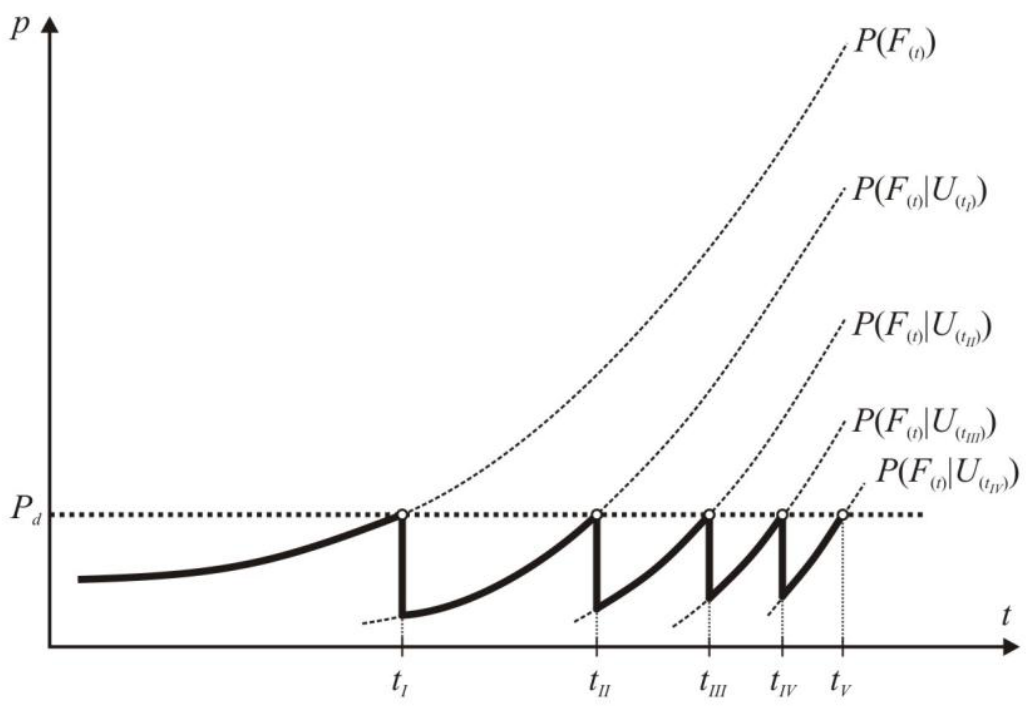

Fig. 3. Probabilities of failure Pf calculated according (7) and (18) with the times of 5 inspections.

\section{Conclusion}

Propagation of the fatigue cracks and possible prediction of such propagation in time since the start of variable loading effects is the case when probabilistic methods must be used because too many uncertainties influence the determination of the input values.

The computation uses the newly developed Direct Optimized Probabilistic Calculation ("DOProC"), which appears to be a very efficient tool to make probabilistic assessment of the structural reliability on the basis of the exact definition of the acceptable size of the fatigue crack.

The DOProC method and its application in probabilistic prediction of fatigue crack damage can considerably improve estimation of maintenance costs for the structures and bridges subject to cyclic loads. If this methodology is developed further, the goal of investigations seems to be, in particular, application of Bayesian networks in the computational model $[12,13]$, which describes propagation of fatigue cracks in the system. 
This contribution has been developed as a part of the research project GACR 17-01589S "Advanced computational and probabilistic modelling of steel structures taking account fatigue damage" supported by the Czech Grant Agency and also has been completed thanks to the financial support provided to VSB-Technical University of Ostrava by the Czech Ministry of Education, Youth and Sports from the budget for conceptual development of science, research and innovations for the 2017 year.

\section{References}

1. K. Kotrasova, I. Grajciar, E. Kormanikova, A case study on the seismic behavior of tanks considering soil-structure-fluid interaction, Journal of Vibrational Engineering and Technologies 3, 3, 315-330 (2015)

2. J. Kralik, J. Kralik jr., Failure probability of NPP communication bridge under the extreme loads, Applied Mechanics and Materials 617, 81-85 (2014) DOI: 10.4028/www.scientific.net/AMM.617.81.

3. P. Janas, M. Krejsa, V. Krejsa, Př́mý Optimalizovaný Pravděpodobnostní Výpočet Direct Opzimized Probabilistic Calculation, 1st ed. (VSB-Technical University of Ostrava, 2015, in Czech)

4. M. Krejsa, P. Janas, V. Krejsa, Structural Reliability Analysis Using DOProC Method, Procedia Engineering 142, 34-41 (2016). DOI: 10.1016/j.proeng.2016.02.010

5. M. Krejsa, L. Koubova, J. Flodr, J. Protivinsky, Q.T. Nguyen, Probabilistic prediction of fatigue damage based on linear fracture mechanics, Frattura ed Integrita Strutturale 11, 39, 143-159 (2017). DOI: 10.3221/IGF-ESIS.39.15

6. M. Krejsa, Z. Kala, S. Seitl, Inspection based probabilistic modeling of fatigue crack progression, Procedia Engineering 142, 145-152, (2016). DOI: 10.1016/j.proeng.2016.02.025

7. R. Halama, M. Fusek, Z. Poruba, Influence of mean stress and stress amplitude on uniaxial and biaxial ratcheting of ST52 steel and its prediction by the AbdelKarimOhno model, International Journal of Fatigue 91, 313-321, (2016). DOI: $10.1016 /$ j.ijfatigue.2016.04.033

8. S. Seitl, T. Thienpont, W. De Corte, Fatigue crack behaviour: comparing three-point bend test and wedge splitting test data on vibrated concrete using Paris' law, Frattura ed Integrita Strutturale 39, 110-117, (2017). DOI: 10.3221/IGF-ESIS.39.12

9. I. Major, M. Major, Traveling waves in a thin layer composed of nonlinear hyperelastic zahorski's material, Journal of Theoretical and Applied Mechanics 47, 1, 109-126, (2009)

10. M. Krejsa, V. Tomica, Determination of Inspections of Structures Subject to Fatigue, Transactions of the VSB - Technical University of Ostrava, Civil Engineering Series 11, 1, 1-9, (2011). DOI: 10.2478/v10160-011-0007-x

11. J. Vican, J. Gocal, J. Odrobinak, M. Moravcik, P. Kotes, Determination of railway bridges loading capacity, Procedia Engineering 111, 839-844, (2015). DOI: 10.1016/j.proeng.2015.07.155

12. S. Mahadevan, R. Zhang, N. Smith, Bayesian networks for system reliability reassessment, Struct. Saf. 23, 231-251, (2001). DOI: 10.1016/S0167-4730(01)00017-0

13. J. Luque, D. Straub, Reliability analysis and updating of deteriorating systems with dynamic Bayesian networks, Struct. Saf. 62, 34-46, (2016). DOI: $10.1016 /$ j.strusafe.2016.03.004 